\title{
Fatigue-induced changes of impedance and performance in target tracking
}

\author{
L. P. J. Selen · P. J. Beek $\cdot$ J. H. van Dieën
}

Received: 1 December 2006/ Accepted: 11 February 2007/Published online: 7 March 2007

(C) Springer-Verlag 2007

\begin{abstract}
Kinematic variability is caused, in part, by force fluctuations. It has been shown empirically and numerically that the effects of force fluctuations on kinematics can be suppressed by increasing joint impedance. Given that force variability increases with muscular fatigue, we hypothesized that joint impedance would increase with fatigue to retain a prescribed accuracy level. To test this hypothesis, subjects tracked a target by elbow flexion and extension both with fatigued and unfatigued elbow flexor and extensor muscles. Joint impedance was estimated from controlled perturbations to the elbow. Contrary to the hypothesis, elbow impedance decreased, whereas performance, expressed as the time-on-target, was unaffected by fatigue. Further analysis of the data revealed that subjects changed their control strategy with increasing fatigue. Although their overall kinematic variability increased, task performance was retained by staying closer to the center of the target when fatigued. In conclusion, the present study reveals a limitation of impedance modulation in the control of movement variability.
\end{abstract}

L. P. J. Selen · P. J. Beek · J. H. van Dieën

Institute for Fundamental and Clinical Human Movement

Sciences, Faculty of Human Movement Sciences,

VU University Amsterdam, Van der Boechorststraat 9,

1081 BT Amsterdam, The Netherlands

Present Address:

L. P. J. Selen $(\square)$

Sensorimotor Control Group,

Computational and Biological Learning Lab,

Department of Engineering, University of Cambridge,

Trumpington Street, Cambridge CB2 1PZ, UK

e-mail: Lpjs2@eng.cam.ac.uk

\section{Introduction}

Prolonged exercise induces muscle fatigue. Muscle fatigue is generally defined as an activity induced loss of the ability to produce force with a muscle or muscle group due to a combination of central and peripheral processes (Gandevia 2001). The central nervous system (CNS) can compensate for a loss in the force generating capacity of the individual motor units (peripheral fatigue) by increasing the central drive to the muscle(s), resulting in higher firing frequencies of already active motor units and additional recruitment of larger motor units. Muscle fatigue eventually results in task failure (Hunter et al. 2002, 2004).

In the present study, we were interested in how fatigue influences the variability of motor performance prior to task failure. Motor output variability is, at least in part, caused by variability in muscular force output. Besides the effort required to generate a constant force output under fatigue, also the variability of the force output increases with fatigue (e.g., Lippold 1981; Lorist et al. 2002; Hunter et al. 2004; Huang et al. 2006). Most tasks constrain the range of permissible positional variations and therefore the CNS has to control the effects of force variability on the overt kinematics.

The neuromotor noise theory (NNT, Van Galen and Schomaker 1995) states that in order to obtain a desired level of positional accuracy of the end-effector (e.g., hand, finger, mouse), the effects of force variability are filtered by increasing joint stiffness through muscular coactivation. Most empirical support for the use of impedance to filter neuromuscular noise is based on indirect evidence, such as increased pen tip pressure in writing (e.g., Van Den Heuvel et al. 1998) and on EMG increases with increasing precision demands (e.g., Gribble et al. 2003; Osu et al. 2004). In recent studies, we provided 
direct evidence that individuals increase joint stiffness in order to attain the prevailing accuracy demands (Selen et al. 2006a, b). This modulation of joint stiffness is particularly evident in target tracking.

As an extension of the NNT, Van Dieën et al. (2003) argued that an increase in neural and motor noise with fatigue might necessitate increased co-activation to attain the desired accuracy. Increased co-activation, on the other hand, would further accelerate fatigue development, resulting in a vicious circle of fatigue development and muscular co-activation under strict task constraints. Indications of increased co-activation with fatigue have been found (Gagnon et al. 1992; Psek and Cafarelli 1993), but given that the EMG-force relationship is affected by fatigue, this provides only tentative support.

Apart from an increase in force variability with fatigue, also the muscle impedance changes with fatigue. With fatigue, muscle stiffness decreases for a constant isometric force, whereas the damping increases (Zhang and Rymer 2001). This would exacerbate the vicious circle identified by Van Dieën et al. (2003) because a disproportionate increase in activation would be needed to generate satisfactory stiffness.

In previous studies, we not only found that elbow impedance changed with accuracy demand, but also that subjects tend to adapt their movement speed within the margins imposed by the task. For time-constrained goal directed movements, this resulted in a small but significant decrease of movement velocity with smaller targets (Selen et al. 2006a). During target tracking, where movement velocity is more severely constrained, subjects changed the organization of corrective movements (Selen et al. 2006b).

In the present study, we aimed to further extend our understanding of the control of joint impedance in relation to motor output variability. Following our previous manipulations of accuracy demand, we now manipulated the neuromuscular noise by inducing muscular fatigue. We hypothesized that joint impedance would increase in order to compensate for the fatigue-induced neuromuscular noise and concomitant kinematic variability.

\section{Methods}

\section{Subjects}

Ten healthy subjects (five males and five females) between 20 and 25 years of age participated in the experiment. All subjects had normal or corrected to normal vision and reported no history of neuromuscular disorders. All subjects were right handed, in the sense that this was the hand they normally used for writing. The Local Ethics Committee approved the experiment and all subjects signed informed consent forms prior to their participation.

Overview of the experiment

The subjects performed two tasks. In the first task, they tracked a sinusoidally moving target (Fig. 1a) by making elbow flexion and extension movements in the horizontal plane. The second task was intended to induce muscular fatigue (Fig. 1b) and consisted of resisting a time varying motor torque. Figure 1c depicts an overview of the order in which both tasks were performed. First, four 2-min unfatigued tracking trials (UFT) were performed. Second, the fatigue protocol (FP) alternated with, now fatigued, tracking (FT). To control for learning effects, the tracking task was repeated for five of the ten subjects after a recovery period of $5 \mathrm{~min}$ (PFT, post fatigue tracking). After every tracking period and every fatigue protocol, subjects were asked to rate their perceived exertion (RPE) in the arm on a ten-point Borg scale (Borg 1982).

Prior to the actual experiment, estimates of maximum voluntary torque, limb inertia and maximum voluntary stiffness were obtained. In the following sections, the experimental setup and procedure will be explained in more detail.

\section{Experimental setup}

Subjects were seated on a chair in front of a semicircular array of light emitting diodes (LEDs). Two permanently active LEDs indicated the target boundaries. The forearm used for writing was tightly cast onto the vertical shaft of a torque controlled motor (S-motor, elu93028, Fokker Control Systems), with the medial epicondyle aligned with the axis of rotation and the palm of the hand facing downwards. A laser pointer was attached to the forearm, indicating the pointing position on the LED array that was $1.5 \mathrm{~m}$ away from the hand. Both torque and position were stored at $1 \mathrm{kHz}$. For a more detailed description and a picture of the experimental setup see Selen et al. (2006a, b).

\section{Experimental procedure}

\section{Maximum voluntary torque assessment}

The torque level of the fatigue protocol was based on an estimate of the maximum voluntary torque (MVT) of the elbow. A force transducer was attached $30 \mathrm{~cm}$ distal to the elbow joint, orthogonal to the cast forearm. Three MVT attempts in both flexion and extension direction were performed, alternated with $1 \mathrm{~min}$ rest. The maximum value, out of six, was selected as the MVT. 

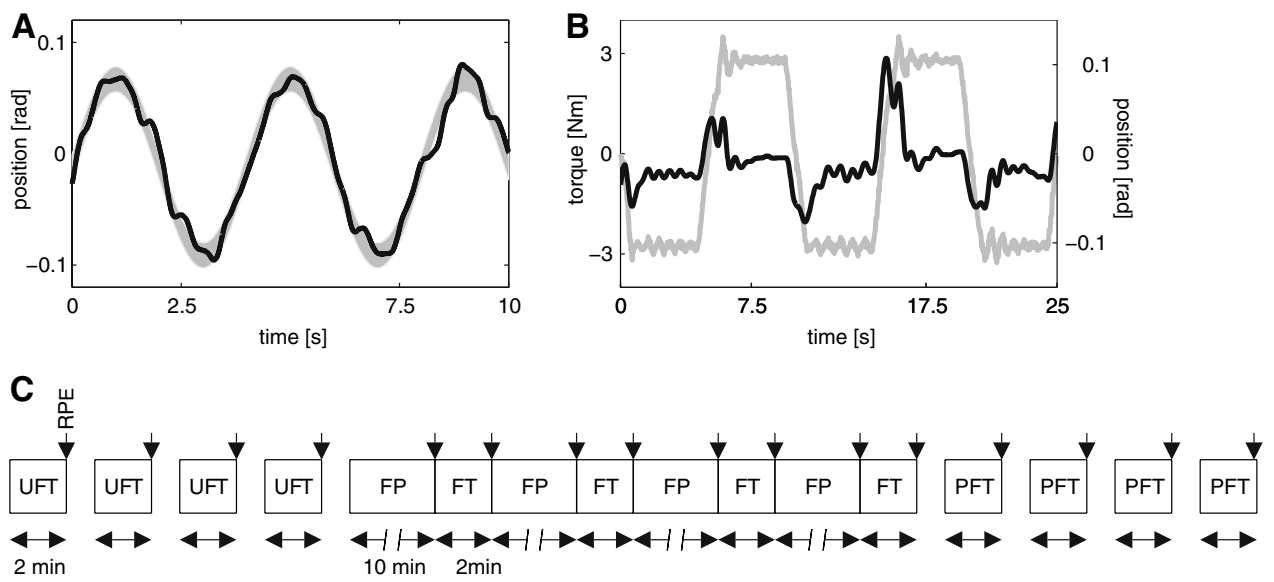

Fig. 1 Schematic description of the experiment. a Section of a tracking trial. The gray area indicates the target area and the black line the projection of the pointer position. b Section of the fatigue protocol. Time varying torque is indicated in gray and the elbow angle in black. c Time line of the experiment. Unfatigued tracking (UFT) and post fatigue tracking (PFT) trials were all followed by 1-min rest. The fatigue protocol (FP) was followed immediately by fatigued tracking (FT). After the last UFT trial and after the last FT trial, subjects had a 5-min rest period. Ratings of perceived exertion were assessed immediately after UFT, FP, FT and PFT and are indicated by the vertical arrows

over the four 2-min trials with the restriction that they had to be at least $5 \mathrm{~s}$ apart. All perturbations occurred in the zero crossing of the target sine wave, i.e., at maximum velocity. Perturbations were bi-phasic, had an amplitude of $5 \mathrm{Nm}$ and a total duration of $70 \mathrm{~ms}$.

\section{Fatigue protocol}

Fatigue was induced by counteracting a torque generated by the motor (see Fig. 1b). This positional task is believed to generate fatigue much faster than an isometric force production task (Hunter et al. 2004). The time varying motor torque was constant for $4 \mathrm{~s}$ and changed sign in $1 \mathrm{~s}$. Peak values of the torque were 5\% MVT. Subjects opposed this torque pattern for $10 \mathrm{~min}$, immediately followed by FT.

\section{Fatigued tracking}

During UFT, FT and PFT, subjects tracked a target that had a width of $0.027 \mathrm{rad}$ (which is approximately $1^{\circ}$ ) and oscillated at $0.25 \mathrm{~Hz}$ with an amplitude of $0.2 \mathrm{rad}$ (see Fig. 1a). Subjects were instructed to keep the projection of the laser pointer within the target area. In some cycles, the motor applied a bi-phasic torque to the forearm. Subjects were instructed not to intervene voluntarily with those torque perturbations. From these perturbations, estimates of elbow stiffness and damping were calculated.

Two perturbation types were used and each was applied 12 times for every experimental condition. Perturbations were applied during flexion (pFLEX) and extension (pEXT) and started with a torque opposite to the movement direction. The 24 perturbations were distributed randomly
The fatigued tracking task was exactly the same as in UFT. Subjects tracked the target immediately after the fatigue protocol for 2 min, during which six randomly distributed perturbations were applied. The combination of FP and FT was repeated four times.

\section{Post-fatigue tracking}

For five of the ten subjects, the UFT protocol was repeated after the FT protocol. These data were analyzed to test for learning effects and retention in both performance and impedance. 
Analyses

\section{Tracking performance}

The instruction to the subjects was to keep the pointer between the target boundaries. Fulfillment of this performance constraint was quantified as the percentage of time that the pointer was on target (\%TT). In order to reveal changes in control, also the percentage of samples that lagged (\%LAG) the center of the target was calculated. A sample was marked as 'lagging' when its position minus the target center position times the sign of the target velocity was smaller than zero.

Kinematic variability was assessed by calculating the mean distance to the center of the target (MDT), the RMS value of the distance to the center of the target (RMSDT) and the standard deviation of the distance to the center of the target (SDDT) over the unperturbed tracking cycles. The latter two were calculated as a function of cycle time

$$
\begin{aligned}
\operatorname{RMSDT}(t) & =\sqrt{\frac{1}{n} \sum_{j=1}^{j=n}\left[\varphi_{j}(t)-\phi(t)\right]^{2}} \text { and } \\
\operatorname{SDDT}(t) & =\sqrt{\frac{1}{n} \sum_{j=1}^{j=n}\left[\varphi_{j}(t)-\bar{\varphi}(t)\right]^{2}},
\end{aligned}
$$

where $n$ is the number of unperturbed cycles and $t$ represents time within a cycle. $\phi(t)$ represents the center of the target as a function of time and $\bar{\varphi}(t)$ represents the average position over cycles of the laser as a function of time. Subsequently, the time-averaged value was calculated (RMSDT and SDDT).

\section{Impedance estimation}

The dynamics of the elbow joint were estimated by fitting a second order linear model with stiffness $K$, damping $B$ and inertia $I$ to the kinematic deviations in response to the torque perturbations. The inertia was estimated in a separate step and kept constant over conditions (UFT, FT and PFT) and movement directions (pFLEX and pEXT). The time window for the optimization was $150 \mathrm{~ms}$, avoiding contributions of voluntary responses, but including spinal reflexes to the impedance estimates. After the optimization procedure the variance accounted for (VAF) was calculated. For a detailed description of the optimization procedure, see Selen et al. (2006a, b).

\section{Characterization of submovements}

Tracking movements are composed of small submovements. These submovements are best visible in the velocity domain where they appear as speed pulses (SP) (see Roitman et al. 2004; Selen et al. 2006b). To quantify SPs, angular data were filtered with a fifth order Butterworth filter with a cut-off frequency of $6 \mathrm{~Hz}$ and subsequently numerically differentiated. The duration of a single SP (SP duration) was defined as the time between two successive local minima in the velocity profile. The amplitude of an SP (SP amplitude) was defined as the difference between a local maximum in the velocity profile and the average value of the two nearest minima. The slope of the linear regression between SP duration and SP amplitude was interpreted as an error correction gain (SP gain). SP gain has been shown to increase with increasing movement velocity (Roitman et al. 2004; Selen et al. 2006b) and increasing accuracy demand (Selen et al. 2006b).

\section{Statistics}

Both in the text and figures data will be presented as mean and SD. In the figures, the four separate trials per condition will be presented. However, to remove learning effects, statistics will be presented for the last two trials only. Unless mentioned otherwise, there was no difference with the statistics as determined for all four trials. Statistics for stiffness and damping will be presented for all four trials together.

The focus will be on the difference between UFT and FT. The effects of fatigue were examined by performing two-way (two or four trials $\times$ two conditions) repeated measures ANOVAs on all ten subjects. Additional two-way (two trials $\times$ conditions) repeated measures ANOVAs were performed on the subgroup of five subjects that also performed PFT. For the stiffness and damping two-way (two movement directions $\times$ two conditions) ANOVAs were performed. Honest significant difference (HSD) Tukey's tests were used to further analyze significant effects.

\section{Results}

Fatigue

All subjects completed the four repetitions of the $10 \mathrm{~min}$ fatigue protocol. Figure 2 presents the summary of their RPE scores. Subjects reported increased exertion due to the FP, which slightly decreased during FT but remained above baseline level of unfatigued tracking. During PFT, RPE scores had returned to UFT levels.

\section{Tracking performance}

Measures of tracking performance are depicted in Fig. 3 itemized for the four 2-min trials per condition. The sta- 
tistics related to UFT and FT as calculated for the final two trials per condition are presented in Table 1. At first sight, the results appear to indicate an increase of \% TT over time. However, no significant effect of trial number was found for any performance measure, either with all four trials or with only the last two trials included.

\% TT is the performance measure that corresponds with the instruction to the subjects to stay on target. Despite the fatigue protocol, no changes in tracking performance were observed. However, fatigue resulted in larger kinematic fluctuations (SDDT). Without changes in control this would have resulted in lower \% TT. The decrease in MDT and \%LAG with fatigue indicates that subjects changed their control strategy to stay closer to the center of the target.

Separate repeated measures ANOVAs were performed for the four subjects that performed PFT in addition to UFT and FT. For \%LAG, MDT, SDDM and RMSDT $P$-values were smaller than 0.02 . The effect of condition on the stiffness estimate $K$ was nearly significant $(P=0.056)$. Figure 4 shows the mean data over those five subjects for all performance variables and the stiffness. All $P$-values smaller than 0.05 in the post-hoc tests are presented. Again, no differences in \% TT were revealed between the three conditions. Both \% LAG and MDT seemed to stay at their FT values, whereas SDDT and RMSDT returned to their baseline, UFT, values during PFT.

Additional analyses revealed that performance variables became much less variable with fatigue. The left panel of Fig. 5 shows the average value of performance measures split into eight 1-min sections for a single subject. The right

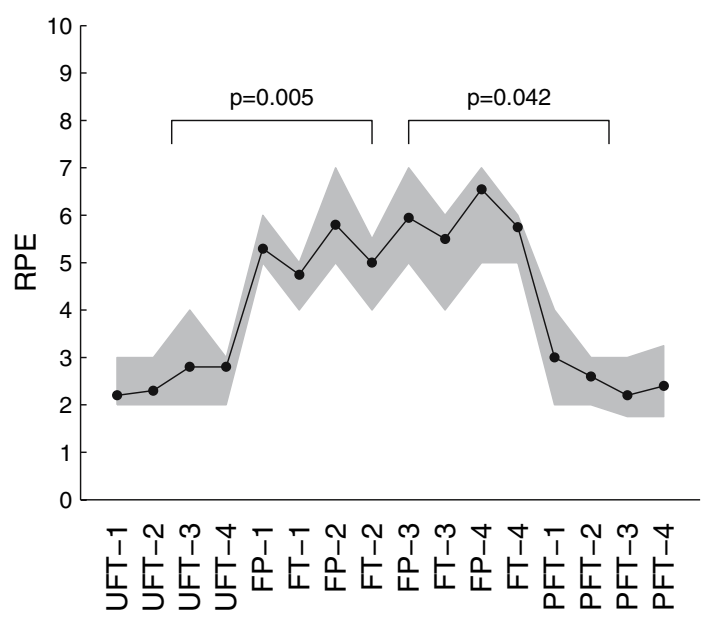

Fig. 2 Mean ratings of perceived exertion (RPE) over the entire experimental protocol. The gray area indicates $\pm 1 \mathrm{SD}$. Ratings were obtained immediately after un-fatigued tracking (UFT), the fatigue protocol (FP), fatigued tracking (FT) and post-fatigue tracking (PFT). RPE scores after FT were used for statistical comparisons between conditions panel shows the mean and SD over all subjects of the standard deviation over the 8 min sections. It is evident that performance was less variable between trials in the fatigued condition.

\section{Impedance}

The impedance of the elbow joint was estimated by fitting a $K-B-I$ model to the experimental data. The inertia was estimated independently of $K$ and $B$ and ranged from 0.047 to $0.0833 \mathrm{Nms}^{2} / \mathrm{rad}$. Maximum voluntary stiffness was 61 (SD 17) Nm/rad and maximum voluntary damping was 0.81 (SD 0.22) Nms/rad. Figure 6 presents the impedance
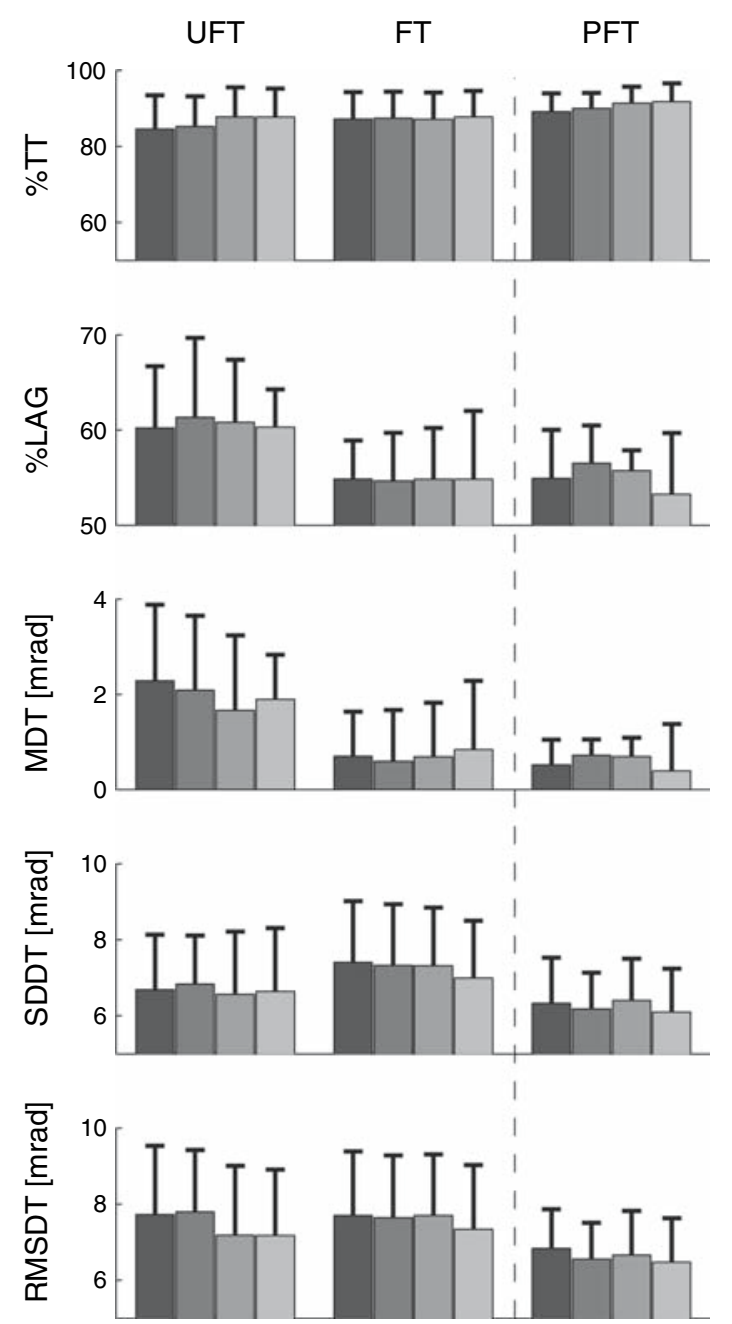

Fig. 3 Mean and SD over subjects of tracking performance measures, itemized for the four repeated trials (dark to light bars) and the three experimental conditions [un-fatigued tracking (UFT), fatigued tracking (FT), post fatigue tracking (PFT)]. Note that for UFT and FT the results are based on ten subjects and for PFT on five subjects. This is indicated by the vertical dashed line. See main text for explanation of the performance variables 
Table 1 Statistical effects of the physical state of the subjects (State) and trial number (Trial) on performance measures

\begin{tabular}{|c|c|c|c|}
\hline & $F_{(1,9)}$ & $P$ & $\eta_{\mathrm{p}}^{2}$ \\
\hline \multicolumn{4}{|l|}{$\% \mathrm{TT}$} \\
\hline State & 0.062 & 0.809 & 0.007 \\
\hline Trial & 0.000 & 0.289 & 0.124 \\
\hline State $\times$ Trial & 0.000 & 0.434 & 0.069 \\
\hline \multicolumn{4}{|l|}{$\%$ LAG } \\
\hline State & 14.878 & $0.004 *$ & 0.623 \\
\hline Trial & 0.000 & 0.810 & 0.007 \\
\hline State $\times$ Trial & 0.000 & 0.819 & 0.006 \\
\hline \multicolumn{4}{|l|}{ MDT } \\
\hline State & 9.596 & $0.013 *$ & 0.516 \\
\hline Trial & 0.401 & 0.541 & 0.043 \\
\hline State $\times$ Trial & 0.019 & 0.892 & 0.002 \\
\hline \multicolumn{4}{|l|}{ SDDT } \\
\hline State & 0.576 & $0.040 *$ & 0.390 \\
\hline Trial & 2.519 & 0.447 & 0.219 \\
\hline State $\times$ Trial & 4.669 & 0.614 & 0.064 \\
\hline \multicolumn{4}{|l|}{ RMSDT } \\
\hline State & 0.428 & 0.530 & 0.045 \\
\hline Trial & 3.344 & 0.072 & 0.271 \\
\hline State $\times$ Trial & 1.112 & 0.351 & 0.110 \\
\hline
\end{tabular}

States are unfatigued (UFT) and fatigued (FT). Trials are the four 2-min tracking periods. Partial- $\eta^{2}\left(\eta_{\mathrm{p}}^{2}\right)$ is presented as a measure of effect size

*Indicates that the result was significant $(P<0.05)$
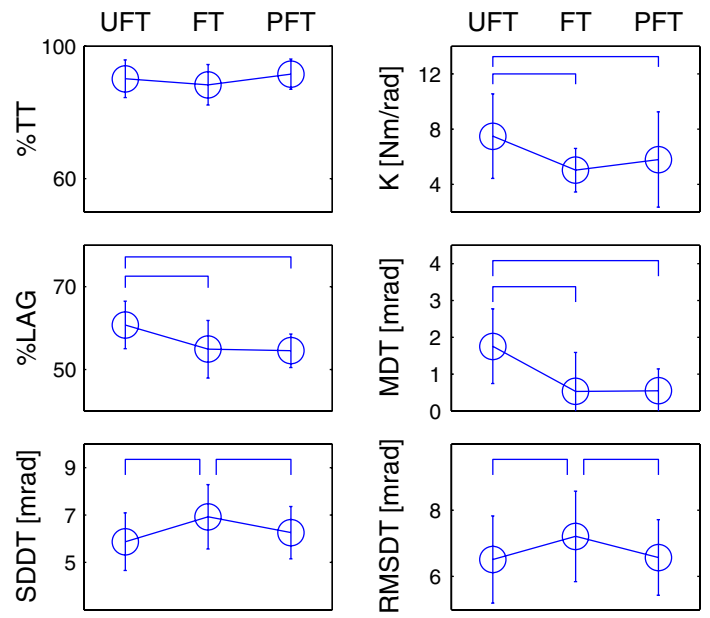

Fig. 4 Post fatigue tracking (PFT) performance. Five subjects performed an additional PFT task. Repeated measures ANOVAs were executed for the mean of the performance variables in the last two trials of each condition. Stiffness values were averaged over movement directions. Overlines indicate $P$-values smaller than 0.05

estimates, expressed as stiffness and damping. The fatigue protocol resulted in smaller stiffness values $\left[F_{(1,9)}=\right.$ 24.238, $\left.P=0.001, \eta_{\mathrm{p}}^{2}=0.729\right]$ compared to UFT values.
During PFT the stiffness recovered slowly, but not significantly (Fig. 4). Damping estimates did not change in response to fatigue $\left[F_{(1,9)}=0.634, P=0.449\right]$. In all cases the VAF was higher than 0.9 .

Apart from fatigue, the stiffness estimate was also affected by perturbation direction. The stiffness was higher during extension than during flexion $\left[F_{(1,9)}=30.474\right.$, $\left.P<0.000, \eta_{\mathrm{p}}^{2}=0.772\right]$.

Speed pulses

Speed pulses were extracted from the speed profiles of the unperturbed movement cycles. Their characteristics (SP amplitude, SP duration and SP gain) are depicted in Fig. 7 for the individual trials and conditions. After a strong learning effect in the first two trials of UFT $\left[F_{(1,9)}=10.018\right.$, $P=0.000, \eta_{p}^{2}=0.590$ for SP gain], no differences in the organization of speed pulses were observed between the unfatigued and fatigued condition for the remaining trials.

\section{Discussion}

In the present study we tested the hypothesis that fatigueinduced increase of force variability would result in increased elbow impedance in order to retain the accuracy required by the task. Based on \% TT, no effects of fatigue on task performance were revealed. Contrary to our hypothesis, however, this was not accompanied by an increase in elbow impedance (i.e., stiffness), suggesting that the vicious circle between fatigue development and muscular co-activation as proposed by Van Dieën et al. (2003) does not exist or is circumvented. This is supported by the finding that muscular activity does not increase in response to fatigue in multi-joint target tracking (Huysmans et al. 2007).

In the following sections, we will first discuss why joint impedance did not increase and how other mechanisms might have contributed to preserving task performance in the face of fatigue-induced increased neuromuscular noise. Subsequently, we will explain the results in view of reduced solution spaces and explorative behavior. Finally, we will draw some general conclusions about the implications of the present findings for the concept of impedance modulation as a generic means to cope with neuromuscular noise.

Before discussing the present results, we have to ascertain that the fatigue protocol was effective. Fatigue was only assessed indirectly by ratings of perceived exertion as previous studies showed that RPE is a good indicator of muscle fatigue (e.g., Kankaanpää et al. 1997; Hummel et al. 2005; Huysmans et al. 2007). As expected, RPE increased in response to the fatigue protocol. Besides 

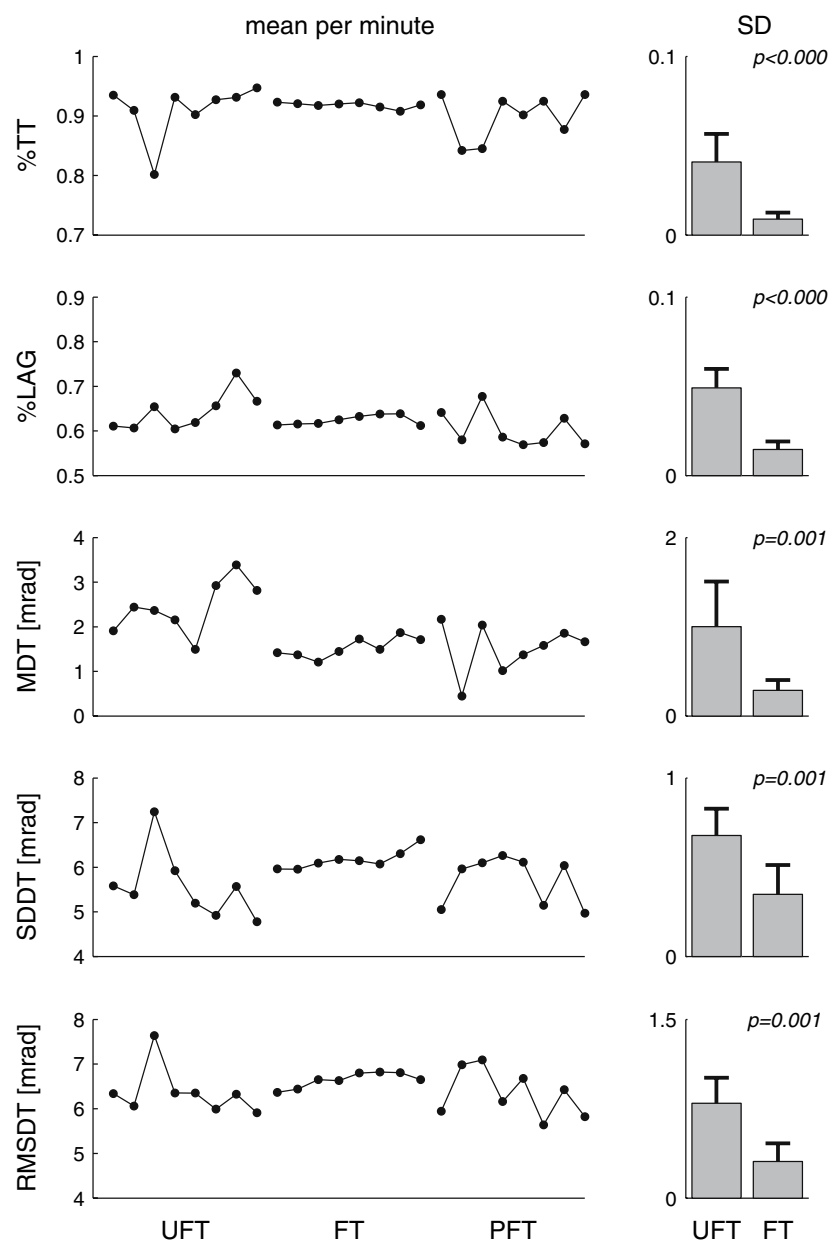

Fig. 5 Control variability. Left column shows the mean performance measures from minute to minute un-fatigued (UFT), fatigued (FT) and post fatigue tracking (PFT) for a typical subject. Right column shows the mean and SD for all subjects of the standard deviation over 8-min for UFT and FT to show that the depicted effect generalized across subjects. Statistical significance is also indicated. First row: percentage time-on-target (\%TT), second row: percentage of time that the pointer lagged the center of the target (\%LAG), third row: mean distance to the center of the target (MDT), fourth row: SD of the distance to the center of the target (SDDT), fifth row: RMS distance to the center of the target (RMSDT)

this expected increase in RPE, the decrease of joint impedance and the increase of SDDT also indicate that the fatigue protocol was effective. The increase in SDDT further indicates increased neuromuscular noise due to fatigue, as reported previously for isometric force (Lippold 1981; Lorist et al. 2002; Hunter et al. 2004; Huang et al. 2006).

Although there was a significant increase in RPE, concomitant with an increase in neuromuscular noise, no decrease in performance was observed. Based on previous studies in which joint impedance was found to increase with increasing accuracy demand (Selen et al. 2006a, b), we expected joint impedance to increase in the present
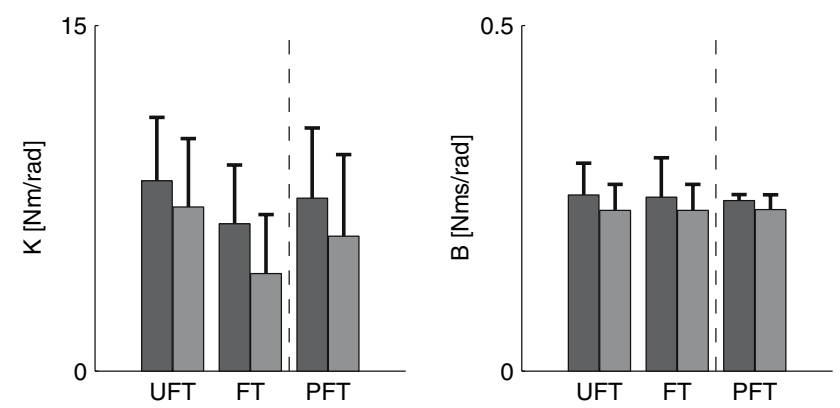

Fig. 6 Mean and SD of stiffness $(K$, left $)$ and damping $(B$, right $)$ for un-fatigued (UFT), fatigued (FT) and post fatigue (PFT) tracking. Left and right bars indicate, respectively, estimates during elbow extension and elbow flexion
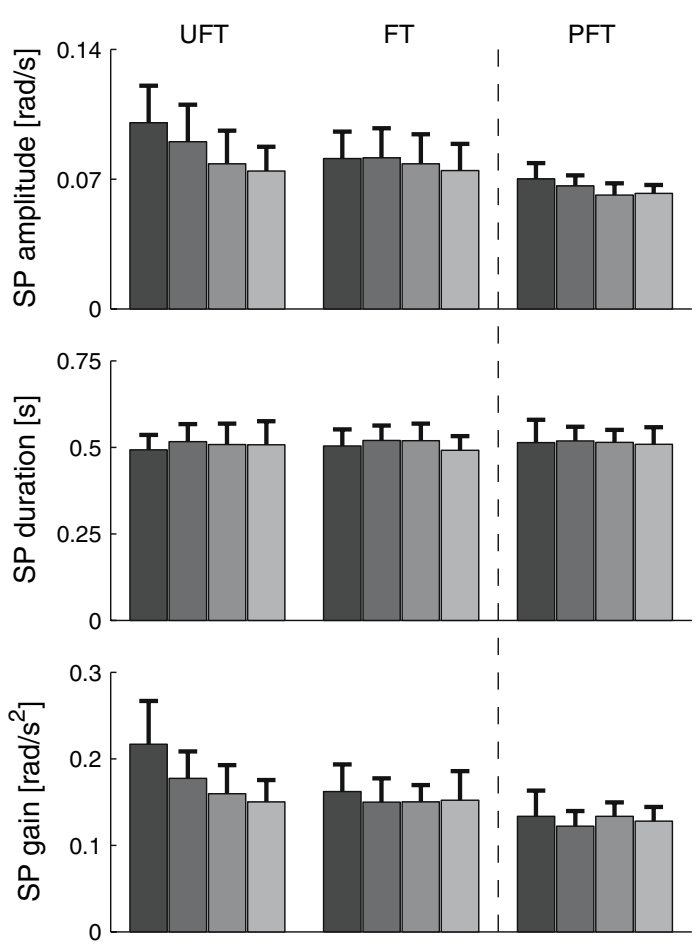

Fig. 7 Mean and SD over subjects of speed pulse characteristics, optimized for the four repeated trials and the three experimental conditions. Note that for un-fatigued tracking (UFT) and fatigued tracking (FT) the results are based on ten subjects and for post fatigue tracking (PFT) on five subjects. This is indicated by the vertical dashed line. See main text for explanation of the speedpulse characteristics

experiment as well. This expectation was based on the consideration that increasing the accuracy demand and increasing the noise are equivalent in that they both require adaptations from the neural control system to increase the signal-to-noise ratio. Nevertheless, joint stiffness decreased by $30 \%$ (SD 19\%).

The applied impedance estimation method does not allow separating out the muscle intrinsic and reflexive con- 
tributions to the fatigue-induced impedance changes. Based on previous system identification studies of the isometric arm under fatigue, we expected that both muscle intrinsic stiffness and reflex contributions decrease with fatigue (Zhang and Rymer 2001).

Could it have been the case that a further increase of impedance was impossible under the prevailing task conditions? We suspect not. Prior to fatigue, stiffness levels were relatively low (about $10 \%$ of their maximum). Due to fatigue the maximum stiffness decreases to about $60 \%$ (Zhang and Rymer 2001). This leaves enough margin to increase joint impedance, although excessive muscular coactivation would be necessary, and renders it unlikely that subjects were unable to increase impedance.

What then prevented the neural control system from increasing joint impedance? Was it some kind of cost consideration or was an impedance strategy no longer adequate to serve the task goal when fatigued? Cost considerations might have played a role. First, just to reach the stiffness level of UFT, co-activation has to increase at least $100 \%$ during FT (Zhang and Rymer 2001). This is much more than the increase in stiffness of up to $30 \%$ we found in previous studies involving a threefold increase in accuracy demand (Selen et al. 2006a, b). Second, the control system may have learned to circumvent the vicious circle associated with impedance modulation (Van Dieën et al. 2003). On the other hand, it is conceivable that impedance modulation is no longer an adequate strategy when fatigued. The delicate balance between increases in neuromuscular noise and impedance (Selen et al. 2005) with cocontraction may have shifted toward an increase in kinematic variability with increasing impedance. Based on the present data it is impossible to differentiate between the aforementioned possibilities, but it follows from the observed decrease in joint impedance that the neural control system must have relied on some other strategy to compensate for the detrimental effects of increased neuromuscular noise due to fatigue.

What could this strategy be? In principle, the neural control system may have employed both feedback and feedforward control strategies to preserve movement accuracy. Feedback control strategies can be revealed by the analysis of submovements. In a previous study, we observed systematic changes in the control of submovements when tracking targets of different frequency and different width (Selen et al. 2006b). Both higher target frequencies and smaller targets resulted in an increase of SP gain as a result of an increase in SP amplitude. Similarly, Pasalar et al. (2005) reported that SP gain increases for larger external perturbing force fields in circular drawing. All those increases may be interpreted as a result of a feedback controlled (relative) error correction mech- anism. In particular for the external perturbing force field manipulations, parallels may be drawn to fatigue-induced increase in neuromuscular noise as both manipulations imply an increase of the perturbing forces. However, we observed no changes in SP gain, SP amplitude or SP duration with fatigue. Only an initial decrease of SP gain and SP amplitude was observed in the first few unfatigued trials, which was most likely due to adaptation to the prevailing task conditions. Apparently, feedback control did not change in response to fatigue. Instead, it is apparent from the present data that, when sufficiently fatigued, the neural control system adopted a feedforward strategy of staying closer to the center of the target, as evidenced by decreased \%LAG and decreased MDT. The predictability of the sine motion of the target allowed for this feedforward strategy, although in both the unfatigued and fatigued states, subjects lagged behind the target most of the time. Adopting this feedforward strategy is understandable because it allows the task constraints to be retained in spite of the increase in within-trial variability (SDDT and RMSDT).

Besides a strategy change due to fatigue, we observed that the execution of that strategy became stereotyped when fatigued. The within-trial variability of all performance measures decreased as fatigue increased over time. How should we interpret this observation? In all likelihood, the experimental manipulation reduced the solution space in two ways, namely by increasing neuromuscular noise and by changing the biophysical properties of the neuromuscular system. We submit that during UFT the neural control system was exploring the solution space, whereas during FT it was not because this would hamper performance. Further support for this hypothesis comes from the observation that within-trial variability decreased and between-trial variability increased again during PFT (see Fig. 5), indicating that the solution space was again being explored. Interestingly, comparable observations of stereotypical, i.e., less variable, control strategies were made in patients with tardive dyskinesia (Newell et al. 1993) and with patellofemoral pain (Hamill et al. 1999) while standing respectively running. It is conceivable that also those stereotypical motor behaviors were associated with a reduction of the solution space, in this case as a result of an underlying pathology.

The PFT data also suggest that subjects adhered to the strategy they adopted during fatigue and stayed close to the center of the target (\%LAG, MDT), despite the recurrence of explorative behavior. This might either be taken to imply that subjects simply adhered to the newly discovered control strategy, or that the neuromuscular system was not fully recovered from fatigue, preventing them from a switch to their UFT strategy. 
If subjects discovered a new control strategy of staying close to the center of the target, then why had they not discovered and used it prior to fatigue? An impedance strategy is energetically demanding (Franklin et al. 2004) and one might expect the neural control system to select an energy saving strategy from the outset. On the other hand, an impedance strategy is generic and has low control cost. Possibly, subjects would have discovered the strategy of staying close to the target in the long run. However, fatigue appeared to have forced them into this new strategy. Similar instances of not discovering alternative strategies have been reported for goal directed movements in a divergent force field (Osu et al. 2003). When subjects move to a target in a divergent force field that pushes them away from a straight line, they increase endpoint stiffness to overcome the instability. An equally effective strategy would be to move through the force field, by making curved paths, thus learning the dynamics of the field. However, subjects only discover this strategy when explicitly instructed to do so. Alternatively, subjects might have switched back to their UFT strategy in the long term. Although SDDT and RMSDT returned to their baseline values during PFT, joint stiffness did not return to baseline and therefore could not contribute to the control of movement variability.

\section{Conclusions}

Impedance modulation is not the strategy of choice to preserve movement accuracy in the face of muscle fatigue, suggesting that the vicious circle of continuously increasing impedance with fatigue (Van Dieën et al. 2003) does not exist or is circumvented. Instead, subjects make use of the predictability of the target motion and stay closer to the center of the target in the fatigued state than in the unfatigued state, resulting in unaffected task performance despite increased kinematic variability.

\section{References}

Borg GA (1982) Psychophysical bases of perceived exertion. Med Sci Sports Exerc 14:377-381

Franklin DW, So U, Kawato M, Milner TE (2004) Impedance control balances stability with metabolically costly muscle activation. J Neurophysiol 92:3097-3105

Gagnon D, Arsenault AB, Smyth G, Kemp F (1992) Cocontraction changes in muscular fatigue at different levels of isometric contraction. Int J Ind Ergon 9:343-348

Gandevia SC (2001) Spinal and supraspinal factors in human muscle fatigue. Physiol Rev 81:1725-1789

Gribble PL, Mullin LI, Cothros N, Mattar A (2003) Role of cocontraction in arm movement accuracy. J Neurophysiol 89:2396-2405
Hamill J, Van Emmerik RE, Heiderscheit BC, Li L (1999) A dynamical systems approach to lower extremity running injuries. Clin Biomech (Bristol, Avon) 14:297-308

Huang CT, Hwang IS, Huang CC, Young MS (2006) Exertion dependent alternations in force fluctuation and limb acceleration during sustained fatiguing contraction. Eur J Appl Physiol 97:362-371

Hummel A, Laübli T, Pozzo M, Schenk P, Spillmann S, Klipstein A (2005) Relationship between perceived exertion and mean power frequency of the EMG signal from the upper trapezius muscle during isometric shoulder elevation. Eur J Appl Physiol 95:321326

Hunter SK, Rochette DL, Ortega JD, Enoka RM (2002) Task differences with the same load torque alter the endurance time of submaximal fatiguing contractions in humans. J Neurophysiol 88:3087-3096

Hunter SK, Critchlow A, Shin IS, Enoka RM (2004) Fatigability of the elbow flexor muscles for a sustained submaximal contraction is similar in men and women matched for strength. J Appl Physiol 96:195-202

Huysmans MA, Hoozemans MJM, Van der Beek AJ, De Looze MP, Van Dieën JH (2007) Fatigue effects on tracking performance and muscle activity. J Electromyogr Kines. doi:10.1016/j.jelekin.2006.11.003

Kankaanpää M, Taimela S, Webber CL, Airaksinen O, Hänninen O (1997) Lumbar paraspinal muscle fatigability in repetitive isoinertial loading: EMG spectral indices, Borg scale and endurance time. Eur J Appl Physiol Occup Physiol 76:236-242

Lippold O (1981) The tremor in fatigue. In: Porter RW (ed) Human muscle fatigue. Pitman medical, London, pp 234-248

Lorist MM, Kernel D, Meijman TF, Zijdewind I (2002) Motor fatigue and cognitive task performance in humans. J Physiol 545:313319

Newell KM, Van Emmerik REA, Lee D, Sprague RL (1993) On postural variability and stability. Gait Posture 4:225-230

Osu R, Burdet E, Franklin DW, Milner TE, Kawato M (2003) Different mechanisms involved in adaptation to stable and unstable dynamics. J Neurophysiol 90:3255-3269

Osu R, Kamimura N, Iwasaki H, Nakano E, Harris CM, Wada Y, Kawato M (2004) Optimal impedance control for task achievement in the presence of signal-dependent noise. J Neurophysiol 92:1199-1215

Pasalar S, Roitman AV, Ebner TJ (2005) Effects of speeds and force fields on submovements during circular manual tracking in humans. Exp Brain Res 163:214-225

Psek JA, Cafarelli E (1993) Behavior of coactive muscles during fatigue. J Appl Physiol 74:170-175

Roitman AV, Massaquoi SG, Takahashi K, Ebner TJ (2004) Kinematic analysis of manual tracking in monkeys: characterization of movement intermittencies during a circular tracking task. J Neurophysiol 91:901-911

Selen LPJ, Beek PJ, Van Dieën JH (2005) Can co-activation reduce kinematic variability? A simulation study. Biol Cybern 93:373381

Selen LPJ, Beek PJ, Van Dieën JH (2006a) Impedance is modulated to meet accuracy demands during goal-directed arm movements. Exp Brain Res 172:129-138

Selen LPJ, Van Dieën JH, Beek PJ (2006b) Impedance modulation and feedback corrections in tracking targets of variable size and frequency. J Neurophysiol 96:2750-2759

Van Den Heuvel CE, Van Galen GP, Teulings HL, Van Gemmert AW (1998) Axial pen force increases with processing demands in handwriting. Acta Psychol (Amst) 100:145-159

Van Dieën JH, Visser B, Hermans V (2003) The contribution of taskrelated biomechanical constraints to the development of workrelated myalgia. In: Johansen $\mathrm{H}$, Windhorst U, Djupsjöbacka M, 
Passatore M (eds) Chronic work-related myalgia: neuromuscular mechanisms behind work-related chronic muscle pain syndromes. Gävle University Press, pp 83-93

Van Galen GP, Schomaker WP (1995) Fitts' law as the outcome of a dynamic noise filtering model of motor control. Hum Mov Sci 12:539-571
Zhang LQ, Rymer WZ (2001) Reflex and intrinsic changes induced by fatigue of human elbow extensor muscles. J Neurophysiol 86:1086-1094 